\title{
TOMMI - Deutscher Kindersoftwarepreis 2020: Die Sieger 2020 stehen fest
}

https://doi.org/10.1515/bd-2021-0004

Geschafft! Fast 900 Kinder haben in deutschlandweit rund 20 Bibliotheken unter strengen Sicherheitsmaßnahmen und mit durchdachtem Hygienekonzept die von einer Fachjury nominierten Spiele und Bildungssoftware getestet. In diesem Jahr wurden die Sieger am 17. Oktober erstmals live bei KiKA und auf kika.de ausgezeichnet. Die ersten Plätze in den verschiedenen Kategorien belegen:

- Konsole: „Ring Fit Adventure“ (Nintendo),

- PC: „Spongebob Square Pants - Battle for Bikini Bottom“ (THQ Nordic),

- Apps: „Der kleine Löschmeister“ (Versicherungskammer Bayern) und

- Elektronisches Spielzeug: „Lego Super Mario Adventure“ (LEGO).

In der neuen Kategorie Bildung räumte „Discovery Tour - Das antike Griechenland" von Ubisoft ab.

\section{Bibliotheken: starke Partner der Kinderjury}

„Dass der TOMMI überhaupt im Corona-Krisenjahr 2020 durchgeführt werden konnte, ist allein der Beherztheit und dem starken Engagement der Mitarbeiter^innen der teilnehmenden Bibliotheken zu verdanken“, erklärt Initiator Thomas Feibel. „Einmal mehr zeigt sich für die Bürger^innen die Verlässlichkeit dieser Bildungshäuser.“

\section{Sonderpreis Kindergarten \& Vorschule // TOMMI Förderpreis Kindergarten Prof. Dr. Stefan Aufenanger, Medienpädagoge an der Universität Mainz, führte den Sonderpreis Kindergarten \& Vorschule durch. Sieger ist die App „Der kleine Löschmeister“. In Zusammenarbeit mit der Auerbach Stiftung wurden erstmals beim neuen TOMMI Förderpreis Kindergarten erprobte und praxistaugliche Kon- zepte zum Einsatz digitaler Medien in Kitas ausgezeichnet. Je 500 Euro Preisgeld erhalten die Kita Hanna vom Kolle (Berlin), die Kita BZ St. Konrad (Ravensburg) und die Kita „kleines ganz groß“ (Rostock).}

\section{Schirmherrschaft und Partner}

Das deutschlandweite Medienprojekt TOMMI steht unter der Schirmherrschaft der Bundesfamilienministerin Dr. Franziska Giffey und wird von FEIBEL.DE Büro für Kindermedien (Berlin) herausgegeben. Partner des Preises sind die 
Auerbach Stiftung, der Deutsche Bibliotheksverband e.V. (dbv), Dein SPIEGEL, Deutschlandfunk Kultur, Familie \& Co, Frankfurter Buchmesse, Google, jugendschutz.net, KiKA, mobilsicher.de, Partner \& Söhne und das ZDF-Kinder- und Jugendprogramm.

\section{Pressekontakt}

\section{FEIBEL.DE Büro für Kindermedien}

Thomas Feibel

Jenaerstr. 15, 10717 Berlin

Tel.: 030 85733030, Mobil: 0172.9319446, E-Mail: tom@feibel.de

URL: http://www.kindersoftwarepreis.de

\section{DIE SIEGER 2020 // Kurzübersicht}

\section{APPS}

- Platz 1: Der kleine Löschmeister (Versicherungskammer Bayern)

- Platz 2: Anton Plus (Solocode)

- Platz 3: Fiete World- Erweiterungen USA und Mexiko (Ahoiii)

\section{BILDUNG}

- $\quad$ Platz 1: Discovery Tour - Das antike Griechenland (Ubisoft)

- Platz 2: Anton Plus (Solocode)

- Platz 3: Booki Hören und Staunen: Musikinstrumente (Tessloff Verlag)

\section{ELEKTRONISCHES SPIELZEUG 2020}

- $\quad$ Platz 1: Lego Super Mario Adventure (LEGO)

- Platz 2: Osmo Genius Starter Set (Tangible Play Inc.)

- Platz 3: Tap it! (HCM KINZEL) 


\section{KONSOLE}

- Platz 1: Ring Fit Adventure (Nintendo)

- Platz 2: Luigi's Mansion 3 (Nintendo)

- Platz 3: Paper Mario: Origami King (Nintendo)

PC

- Platz 1: Spongebob Square Pants - Battle for Bikini Bottom (THQ Nordic)

- Platz 2: Die SIMS 4 - Nachhaltig leben (Electronic Arts)

- Platz 3: Lonely Mountains: Downhill (Thunderful Publishing)

\section{SONDERPREIS KINDERGARTEN UND VORSCHULE 2020}

- App: Der kleine Löschmeister (Versicherungskammer Bayern)

\section{TOMMI FÖRDERPREIS KINDERGARTEN 2020: Beste KITA-Digital-Konzepte}

- Kita Hanna vom Kolle (Berlin)

- Kita BZ St. Konrad (Ravensburg)

- Kita „kleines ganz groß“ (Rostock)

\section{DIE SIEGER 2020 // BEGRÜNDUNGEN Deutscher Kindersoftwarepreis TOMMI - Sieger}

\section{APPS}

Platz 1: Der kleine Löschmeister (Versicherungskammer Bayern)

Das sagt die Kinderjury: „Der kleine Löschmeister“ gewinnt Platz 1 beim TOMMI 2020, weil wir wie die echte Feuerwehr Menschen retten durften. Als Feuerwehrleute haben wir viele Aufgaben bekommen und fuhren durch die ganze Stadt. Wir mussten Türen aufbrechen, Tiere und alte Menschen retten und in Sicherheit bringen. Es war immer etwas los. Wir haben auch gelernt wie 
man eine Ölpfütze beseitigt und haben richtig viele Feuer gelöscht. Es war toll."

Das sagt die Fachjury: „Schnell in die Klamotten der Feuerwehrleute schlüpfen, Brände löschen und Gefahrenquellen beseitigen? Mit dieser App werden Kinder selbst zu kleinen Löschmeistern. Die Bedienung ist ganz einfach, es sind keine Lesekenntnisse nötig und Hilfestellungen zeigen an, was als nächstes zu tun ist. Die App macht großen Spaß und wartet mit vielen verschiedensten spannenden Aufgaben auf. Wissen zum Thema Feuerwehr und zu Brandverhütung wird spielerisch verpackt. Brandheiße Empfehlung!“

Ab 6 Jahre

\section{Platz 2: Anton Plus (Solocode)}

Das sagt die Kinderjury: „,Anton Plus‘ gewinnt beim TOMMI 2020, obwohl es gar kein echtes Spiel ist, uns aber richtig gut beim Lernen unterstützt. Durch das Lösen der verschiedenen Aufgaben sammeln wir Punkte und Münzen, die wir dann für Spiele und so einlösen können. Mit Anton Plus können wir einen schöneren Avatar gestalten und andere Spiele spielen. So macht Lernen nicht nur Spaß, sondern es macht sogar Lust auf Schule.“

Das sagt die Fachjury: „,Anton“ deckt Mathe, Deutsch, Sachunterricht, Musik und Biologie von der 1. bis zur 10. Klasse ab und behandelt die Themen Lehrplan-konform. Der Nutzer kann nicht nur seine eigene Schule in der App wählen, sondern sich auch mit seinem Kameraden vernetzen. Wer fleißig Aufgaben löst (aktuell über 100.000), wird mit Sternchen und Münzen belohnt, mit denen zahllose kleine Spiele ausprobiert werden können. Dies motiviert zu immer neuer Beschäftigung mit der umfangreichen Lern-App.“

Ab 6 Jahre

\section{Platz 3: Fiete World - Erweiterungen USA und Mexiko (Ahoiii)}

Das sagt die Kinderjury: ,,Fiete World - Erweiterungen USA und Mexiko“ gewinnt beim TOMMI 2020, weil wir darin einfach machen konnten, was wir wollten. Es gibt keine Regeln oder Aufgaben. Die Welt ist schön und farbenfroh und macht gute Stimmung. Wir reisten nach Indien, Amerika und Frankreich und Mexiko. Man darf alles anfassen und ausprobieren. Das Spiel ist zwar für kleinere Kinder, uns hat es aber trotzdem genauso Spaß gemacht.“

Das sagt die Fachjury: „Auch in diesem Jahr entführt Fiete sehr junge Spieler^innen in ein kunterbuntes, seitlich scrollbares Wimmelbuch, in dem es unzählige, witzige Details zu entdecken und manipulieren gibt. Und das nicht nur in Fietes ländlicher Heimat am Meer und den drei bereits bekannten Szenarien, sondern seit Kurzem auch in Mexiko und den USA. So wird der entdeckungsreiche Spaß um Burgerbraterei, Wrestling-Ring und Tag-der-Toten- 
Deko bereichert - und nebenbei auch um sehr viel wunderbar stimmungsvolle Musik.“

Ab 4 Jahre

\section{BILDUNG}

\section{Platz 1: Discovery Tour - Das antike Griechenland (Ubisoft)}

Das sagt die Kinderjury: „,Discovery Tour - Das antike Griechenland‘ gewinnt beim TOMMI 2020 den ersten Platz, weil so ein gutes Programm zum Lernen aussehen soll. Wir gingen auf Touren und erkundeten das ganz alte Griechenland. Es gab keine richtige Geschichte außer der Geschichte von Griechenland. Es war wirklich sehr lehrreich, und die Touren sind sehr anspruchsvoll. Auch die Grafik war toll. Nur schade, dass man nicht kämpfen konnte.“

Das sagt die Fachjury: „Ubisofts lebendes Museum bietet rund 30 interaktive Thementouren durch das antike Griechenland. Vorträge ermöglichen die Begegnung mit Zeitzeugen; Anekdoten, Bildmaterial und kurze Texte machen Leben, Kultur, Politik und historische Ereignisse im antiken Griechenland lebendig. Gelerntes kann am Ende jeder Tour durch ein Quiz überprüft werden. Mankos sind der zum Teil unkritische Umgang mit Quellenmaterial sowie die vergleichsweisen hohen Kosten. Trotzdem ein vielversprechender, neuer Ansatz der Geschichtsdidaktik.“

Ab 10 Jahre

\section{Platz 2: Anton Plus (Solocode)}

Das sagt die Kinderjury: „,Anton Plus‘ gewinnt beim TOMMI 2020, weil es das beste Lernspiel der Welt ist. Es ist sehr gut zum Lernen und dabei übersichtlich und jugendfreundlich gestaltet. Es hat Lerngänge von der 1. bis zur 12. Klasse in vielen verschiedenen Fächern. Wir fanden schon das normale „Anton“ gut, aber mit „Plus“ kann noch mehr freigeschaltet werden. Mit gewonnenen Münzen bekamen wir Spiele freigeschaltet. Diese App war für uns genau passend.“

Das sagt die Fachjury: „,Anton“ ist eine App, die sich ursprünglich hauptsächlich an Grundschüler*innen richtete und eine große Anzahl an Mathe- und Deutschübungen bot. Mittlerweile werden Aufgaben bis in die 10. Klasse und auch Fächer wie Sachunterricht, Biologie, Physik, Musik und DAZ angeboten. ,Anton' zählt ausdrücklich zu den besten derzeit erhältlichen kostenlosen Lernapps für Kinder, auch und gerade weil sie ganz gezielte und weitgehend konzentrierte Lernangebote macht. Einfach und gut.“

Ab 6 Jahre 


\section{Platz 3: Booki Hören und Staunen: Musikinstrumente (Tessloff Verlag)}

Das sagt die Kinderjury: „,Booki Hören und Staunen: Musikinstrumente‘ gewinnt beim TOMMI 2020, weil wir dabei komplett vergessen haben, dass es keinen Bildschirm gibt. Mit dem Buch lernten wir, dass es so viele Musikinstrumente gibt. Und mit dem Hörstift durften wir sie uns alle anhören. Es war auch überhaupt nicht langweilig, weil der Stift auch Lernspiele hat. Schade, dass es sowas nicht als Schulbuch gibt.“

Das sagt die Fachjury: „Woraus besteht eigentlich ein Orchester? Wie klingt eine Trompete? Und wie ist eine Gitarre aufgebaut? Das Buch mit dem zugehörigen Bookii- Stift vermittelt kindgerecht, aber seriös eine Menge Wissen für alle, die sich für Instrumente interessieren. Dazu schaltet man den Booki-Stift nur an, tippt auf eine Stelle im Buch und schon geht's los mit dem Entdecken! Besonders unterhaltsam sind die integrierten kleinen Spiele, in denen Kinder zum Beispiel lernen, die verschiedenen Instrumente am Klang zu unterscheiden.“

Ab 6 Jahre

\section{ELEKTONISCHES SPIELZEUG}

\section{Platz 1: Lego Super Mario Adventure (LEGO)}

Das sagt die Kinderjury: „,Lego Super Mario Adventure‘ gewinnt beim TOMMI 2020 Platz 1, weil damit ein Videospiel ganz echt wird. Schon beim Zusammenbauen hatten wir viel Spaß. Wir haben mit LEGO unsere eigenen Super Mario-Levels gebaut und konnten sie auch immer wieder umbauen. Wenn Super Mario auf die Gegner springt, gibt es Münzen und er kann sprechen. Wir konnten mit LEGO-Steinen ohne App weiterspielen und das hat uns besonders gut gefallen.“

Das sagt die Fachjury: „Erst einmal klingt das Konzept recht schräg: Den Spaß an ,Super Mario in Lego übersetzen. Doch schon nach kurzer Zeit funktioniert das sehr gut. Die Level sind durch markante Teile in die Lego-Welt überführt, Mario selbst ist eine Spielfigur, die Münzen klingeln lässt und sich mit juchzenden Geräuschen durch die Gegend bewegen lässt. Immer wieder kann man die einzelnen Bestandteile neu zusammensetzen und erneut auf Münzenjagd gehen. Für Fans von Lego und Super Mario.“

Ab 7 Jahre

\section{Platz 2: Osmo Genius Starter Set (Tangible Play Inc.)}

Das sagt die Kinderjury: „,Osmo Genius Starter Set‘ gewinnt beim TOMMI 2020, weil wir damit viele verschiedene Spiele spielen konnten. Wir legten Tangram-Steine in bestimmte Muster, mussten verschiedene Wörter erraten und konnten sogar zeichnen. Über den Spiegel sieht Osmo dabei, was wir tun. Von den 
drei Karten von Osmo hat uns das Spiel mit dem farbigen Holzsteinen am meisten Spaß gemacht, weil es total cool ist, wenn man denken muss.“

Das sagt die Fachjury: „Die Idee ist so einfach wie genial: Das iPad wird in eine Vorrichtung eingeklemmt, ein kleines, rotes Element auf die Kamera gesetzt und schon kann es losgehen. Über einen kleinen Spiegel scannt das Tablet dann, was auf dem Tisch abgelegt wird. Die jeweilige App des Starter Sets verbindet dieses immer gleiche Prinzip dann mit unterschiedlichen, unterhaltsamen Spielen. Ob beim klassischen Tangram, beim Rechnen üben oder beim Malen wie ein großer Künstler, an Vielfalt ist das System kaum zu überbieten.“

Ab 5 Jahre

\section{Platz 3: Tap it! (HCM KINZEL)}

Das sagt die Kinderjury: „,Tap it!' gewinnt beim TOMMI 2020, weil es ein sehr gutes Familienspiel ist. Erst drückt einer auf den Buzzer und der Partner muss dann so schnell es geht zurückdrücken. Wer aber zu früh drückt, hat verloren. Es gibt unterschiedliche Modi. Wir haben damit sogar Sport gemacht und mussten viel springen und rennen. Es macht großen Spaß, weil man ein sehr gutes Gedächtnis braucht und schnell reagieren muss.“

Das sagt die Fachjury: „,Tap It‘ ist ein Spiel, bei dem Kinder so schnell wie möglich auf verschiedene Buzzer drücken müssen. Diese kann man mit größtmöglichem Abstand im Zimmer verteilen, so dass zur schnellen Reaktion auch immer noch einiges an Akrobatik dazukommt. Das funktioniert gut und macht Spaß. Wenn nur die grässlichen Töne nicht wären, bei denen man sich an einen Feueralarm erinnert fühlt. Unterhaltsam vor allem mit mehreren Leuten, mehrere Altersgruppen können gut zusammenspielen.“

Ab 7 Jahre

\section{KONSOLE}

\section{Platz 1: Ring Fit Adventure (Nintendo)}

Das sagt die Kinderjury: „,Ring Fit Adventure‘ gewinnt Platz 1 beim TOMMI 2020, weil es beste Spiel war, dass wir je gespielt haben. Wir hielten einen elastischen Ring in der Hand und trugen ein Band am Bein, dann rannten wir in echt los, um gegen Monster zu kämpfen. Uns hat sehr gut gefallen, dass die Level mit viel Sport verknüpft waren und man richtig dabei schwitzt. Das hat uns Spaß gemacht, wir haben keine Sekunde rumgesessen.“

Das sagt die Fachjury: „Bei ,Ring Fit Adventures“ wird mit dem ganzen Körper gespielt. Und nicht nur kleine Fitnessspiele, sondern ein ganzes vollwertiges Abenteuer lang. Mit einem großen Ring und einer Beinschlaufe, an denen die 
Controller der Switch befestigt werden, übersetzt die Konsole die Bewegungen der Spieler*innen in das Spiel. Die Vielseitigkeit ist bemerkenswert: Ring Fit ist für Einzelspieler^innen der beste digitale Trimm-Dich. Und für Freunde und Familie ist es ein super Partyspiel mit unterhaltsamen Casual Games.“

Ab 10 Jahre

\section{Platz 2: Luigi's Mansion 3 (Nintendo)}

Das sagt die Kinderjury: ,,Luigi’s Mansion 3“ gewinnt beim TOMMI 2020, weil es das beste, spannendste und witzigste Horrorspiel ist. Das gruselige Hotel mit den vielen Geistern war mega. Luigi musste sie mit dem Staubsauger besiegen. Die Kämpfe sind sehr cool und du hast sehr schnell einen coolen Fortschritt. Außerdem konnten wir im Laufe des Spiels neue Fähigkeiten freischalten. Die einzelnen Etagen und ihre Schrecken haben uns sehr gefallen.“

Das sagt die Fachjury: „Marios Bruder Luigi muss per Staubsauger ein von Gespenstern übernommenes Hotel reinigen. Die einzelnen Level sind toll gestaltet, bieten viele Möglichkeiten, Geheimnisse zu entdecken und zu probieren, was man alles einsaugen kann. Dazu gibt es coole Bosskämpfe und lustiges Sound-Design. Luigi ist dabei eher schreckhaft, zaudernd und keinesfalls ein großer Held. Zumindest am Anfang nicht. Und damit ist er ein ideales Vorbild für viele Menschen, die auch nicht zum Helden geboren wurden.“

Ab 10 Jahre

\section{Platz 3: Paper Mario: Origami King (Nintendo)}

Das sagt die Kinderjury: ,,Paper Mario: Origami King“ gewinnt beim TOMMI 2020, weil es sehr bunt, fröhlich und süß gestaltet ist und wir nur Positives für das Spiel empfinden. Mit einer spannenden Geschichte werden wir in das Abenteuer entführt und sollen unbedingt verhindern, dass ein fieser König Marios Welt zu Origami faltet. In diesem Spiel mit lustigen Pappfiguren wird gekämpft, gewonnen und verloren und viel Spaß hervorgerufen.“

Das sagt die Fachjury: „Ein königliches Vergnügen: Nintendo zeigt mit ,Paper Mario', was die Switch alles drauf hat. Von einer liebevoll gestalteten Welt über witzige Charaktere, eine spannende Story bis hin zur perfekt passenden Steuerung bietet dieses Spiel alles, was man von einem Super-Mario-Titel erwartet. Selbst der Wiederspielwert dürfte gewaltig sein, weil es immer noch Geheimwege zu entdecken gibt. ,Paper Mario“ ist motivierend und abwechslungsreich, der Schwierigkeitsgrad niemals frustrierend und vor allem unheimlich witzig.“

Ab 8 Jahre 
PC

Platz 1: Spongebob Square Pants - Battle for Bikini Bottom (THQ Nordic)

Das sagt die Kinderjury: „,Spongebob Square Pants - Battle for Bikini Bottom gewinnt beim TOMMI 2020 Platz 1, weil wir finden, dass Arbeit belohnt werden muss. Wir lieben die Serie und wir lieben das Spiel, beide sind so lustig und witzig. Es hat großen Spaß gemacht, Bikini Bottom vor den Robotern zu beschützen. Die Geschichte ist spannend und die Fähigkeiten sehr cool. Es gibt Attacken, dass man sterben kann ist aber doof."

Das sagt die Fachjury: „Ach, SpongeBob macht einfach Spaß. Kaum ein Spiel ist so geeignet, die schlechte Laune zu vertreiben, wie das Abenteuer rund um den Schwammkopf. Das liegt sicherlich auch an der detailgetreuen Umsetzung der Charaktere der Sendung, inklusive Stimmen. Allerdings sind die Rätsel und Mini-Spiele des Adventures nicht immer leicht zu knacken und erfordern einiges an Geduld. Witzige Sprüche und ausgesprochen nette Ideen runden den durchweg positiven Eindruck ab“.

Ab 10 Jahre

\section{Platz 2: Die SIMS 4 - Nachhaltig leben (Electronic Arts)}

Das sagt die Kinderjury: „,Die SIMS 4 - Nachhaltig leben“ gewinnt beim TOMMI 2020, weil sich das Simulationsspiel anfühlt wie das echte Leben und wir hier unser Leben selbst entscheiden dürfen. Wir konnten diesmal nicht nur eine Familie gründen, sondern auch viel über Umweltschutz und Recycling lernen. Wir kamen auf neue Ideen, weil wir zum Beispiel aus alten Sachen neue Dinge machen konnten. Das wollen wir jetzt zu Hause auch ausprobieren.“

Das sagt die Fachjury: „Die SIMS machen jetzt auch auf öko? Ja, und sie machen das sogar ziemlich gut. Deshalb hat das Spiel das Potential, junge Nutzer für das Thema nachhaltiges Leben zu sensibilisieren. Durch Recycling, Sammeln von Regenwasser und der Stromerzeugung durch Solarzellen werden die SIMS zum Selbstversorgerhaushalt. Oder sie verbessern den ökologischen Fußabdruck ihres Stadtteils.“

Ab 10 Jahre

\section{Platz 3: Lonely Mountains: Downhill (Thunderful Publishing)}

Das sagt die Kinderjury: „Lonely Mountains: Downhill‘ gewinnt beim TOMMI 2020, weil die Schwierigkeit einen manchmal echt verzweifeln lässt und das aber trotzdem Spaß macht. Als Radfahrer musst du auf unübersichtlichen Strecken einen steilen Berg hinab in Bestzeit meistern. Fährst du gegen Steine, bist du tot. Fällst du von zu hoch runter, bist du tot. Es lässt sich nicht so leicht durchspielen. Aber man will es einfach trotzdem schaffen, das ist richtig gut gemacht!“ 
Das sagt die Fachjury: „Das Indie-Game ,Lonely Mountains: Downhill‘ schafft es, aus einer gefährlichen Extremsportart ein spaßiges Sportspiel zu machen. Die Aufgabe ist schnell verstanden: Mit dem Rad geht es auf extremen Höhen steil und möglichst heil den Berg hinab. Dabei kommt es auf großes Geschick und gutes Reaktionsvermögen an. Der Anreiz liegt auf dem Grat zwischen Versuchen und Versagen. Die Frustrationstoleranz wird auf eine harte Probe gestellt, der Spielspaß überwiegt aber stets.“

Ab 8 Jahre

\section{SONDERPREIS KINDERGARTEN UND VORSCHULE}

\section{Der kleine Löschmeister (Versicherungskammer Bayern)}

Das sagen die Medienpädagogen: „Mit Hupen und Sirenen die Straßen frei machen und dann mit der Leiter und dem Schlauch Brände an einem Haus löschen: Immer wieder waren die Kinder von den Möglichkeiten begeistert. Sie probierten im Feuerwehrhaus alles aus, was sich animieren ließ und lernten dabei zugleich, wofür Schlauch, Schutzkleidung oder auch Gasmaske gut ist. Zwar wären unterstützende Audiokommentare zu den einzelnen Stationen hilfreich gewesen, aber auch so bekommen Kinder einen guten Einblick, was die Feuerwehr und ihre Männer und Frauen alles machen. Das Spiel ist altersgerecht konzipiert und von seinen Navigationsmöglichkeiten für Kinder übersichtlich. Also alles, was ein gutes Spiel mit pädagogischer Absicht ausmacht.“

\section{TOMMI FÖRDERPREIS KINDERGARTEN 2020: Beste KITA-Digital-Konzepte}

\section{Kita Hanna vom Kolle (Berlin)}

„Frühe Förderung naturwissenschaftlichen Denkens bei Kindern unter Einbezug digitaler als auch analoger Medien steht im Zentrum des pädagogischen Ansatzes der Kita.“

Das sagt die Kita-Fachjury: „In der Kita Hanna vom Kolle in Berlin wird mit Kindern ab vier Jahren in naturwissenschaftlichen Projekten unter Einbezug digitaler Medien geforscht. Projektorientiert kommen das Tablet und das digitale Mikroskop zum Einsatz, mit dem Ziel, die Metamorphose von Raupen zu Schmetterlingen mit zu erleben. Zum Einstieg wurde ein Buch vorgelesen, außerdem wurden Zeichnungen und Fotos angefertigt. Bei Fragen kamen die Kindersuchmaschinen 
,BLINDEKUH“ und ,FragFinn` zum Einsatz. Dieses Zusammenspiel von verschiedenen analogen und digitalen Werkzeugen ist zeitgemäß, spannend und hat verschiedene Kompetenzen der Kinder wie beobachten, zuhören, reden, Wissen austauschen und Verantwortung übernehmen, gestärkt. Projekte wie diese sind für jedes Kind eine große Bereicherung und digitale Lernerfahrung. Das Projekt wird pädagogisch multimedial im wahrsten Sinne des Wortes begleitet, in Gesprächskreisen wird das Erlebte reflektiert und mündlich besprochen. Auch während der Kita-Schließzeiten, bedingt durch die Corona-Pandemie, wurde das Projekt weitergeführt, die Raupen mit nach Hause mitgegeben, und Eltern so instruiert, dass sie mit und ohne digitale Medien das Projekt gemeinsam mit den Kindern fortführen konnten. Damit folgt die Kita der Idee einer frühen Förderung im MINT-Bereich, nutzt digitale Medien darüber hinaus zum digital-vernetzten Lernen.“

\section{Kita BZ St. Konrad (Ravensburg)}

„Das Konzept der Kita verbindet in außergewöhnlich fundierter Weise digitale Technik mit Werten und Persönlichkeit“.

Das sagt die Kita-Fachjury: „Das Medienkonzept der Kita BZ St. Konrad in Ravensburg überzeugt durch seine Botschaft ,Man braucht nicht viel, um viel zu erreichen'. Denn digitale Bildung heißt, nicht nur digitale Technik, sondern zugleich auch Werte und Persönlichkeit zu vermitteln. Das Konzept dieses Kindergartens verbindet beides in außergewöhnlich fundierter Weise. Vor allem die kreativen und gestalterischen Potenziale von Tablets werden in den Vordergrund gestellt. Die Kinder bekommen eine Einführung in dieses Medium und können dann ihren Ideen freien Lauf lassen. Unterstützt werden sie dabei von einer insbesondere für medienpädagogische Projekte ausgebildeten Erzieherin. Sie bindet das Team und die Eltern ein, um schließlich aus einem Vorschulprojekt ein alltagintegriertes, medienpädagogisches Arbeiten entwickelt, welches auf viele andere Einrichtungen übertragbar ist. Auf der inhaltlichen Seite des Ansatzes sind das Anknüpfen an die Lebenswirklichkeit der Kinder, die Verschränkung von analogem und digitalem Arbeiten sowie der verstärkte Einsatz von Bildern und Symbolen in der medienpädagogischen Arbeit positiv hervorzuheben. Dies ermöglicht den Kindern Teilhabe und interessengeleitetes Arbeiten. Wertefragen werden etwa in Bezug auf Verantwortung im Umgang mit den Geräten, aber auch als Respekt gegenüber den anderen Kindern formuliert. Durch die Einbindung medienerfahrener Grundschullehrer*innen kann der medial begleitete Übergang vom Vorschul- zum Grundschulkind gelingen. Diese Zusammenarbeit ist besonders vorbildhaft.“ 


\section{Kita „kleines ganz groß“ (Rostock)}

„Ein sehr gelungenes Projekt, in dem das Verhältnis von Technik, Pädagogik und Kreativität sowie Selbstständigkeit der Kinder sehr gut ausgewogen ist.“

Das sagt die Kita-Fachjury: „Exzellente Ausstattung reicht nicht aus, man muss auch damit etwas anfangen können. Die Kita ,kleines ganz groß' in Rostock setzt digitale, aber auch traditionelle Medien in vielfältige Weise ein. Sie geben den Kindern die Möglichkeit, sich gestalterisch auszudrücken, Erfahrungen zu dokumentieren und kreativ zu sein. Dabei werden alle Sinne angesprochen wie etwa beim Geräusche-Merkspiel oder den produzierten Hörspielen. Zugleich werden Tablets auch genutzt, um Eltern in die medienpädagogische Arbeit der Einrichtung einzubeziehen und sie über die Projekte zu informieren. Auch das Programmieren mit einfachen Anwendungen bekommen die Kinder schon angeboten. All dies ist machbar, da die Kinder auch in die technischen und gestalterischen Möglichkeiten der digitalen Medien sehr gut eingeführt werden. Ebenfalls klug: Die Einbindung der örtlichen Medienvertreter als technische Unterstützer, die gleichzeitig für eine Öffentlichkeit sorgen. Der medienpädagogische Ansatz ist zugleich aber auch kritisch und vermittelt einen kind- und altersgerechten Umgang. Insgesamt handelt es sich um ein sehr gelungenes Projekt, in dem das Verhältnis von Technik, Pädagogik und Kreativität sowie Selbstständigkeit der Kinder sehr gut ausgewogen ist.“ 